\title{
Phylogenetic analysis of bacterial and archaeal species in symptomatic and asymptomatic endodontic infections
}

Correspondence

M. M. Vickerman

mmv4@buffalo.edu

Received 14 July 2006

Accepted 24 August 2006

\author{
M. M. Vickerman, ${ }^{1,2}$ K. A. Brossard, ${ }^{2}$ D. B. Funk, ${ }^{1}$ A. M. Jesionowski ${ }^{2}$ \\ and S. R. Gill ${ }^{2}$ \\ 'Department of Periodontics and Endodontics, University at Buffalo School of Dentistry,
Buffalo, NY, USA \\ ${ }^{2}$ Department of Oral Biology, University at Buffalo School of Dentistry, Buffalo, NY, USA
}

\begin{abstract}
Phylogenetic analysis of bacterial and archaeal 16S rRNA was used to examine polymicrobial communities within infected root canals of 20 symptomatic and 14 asymptomatic patients. Nucleotide sequences from $\sim 750$ clones amplified from each patient group with universal bacterial primers were matched to the Ribosomal Database Project II database. Phylotypes from 37 genera representing Actinobacteria, Bacteroidetes, Firmicutes, Fusobacteria and Proteobacteria were identified. Results were compared to those obtained with species-specific primers designed to detect Prevotella intermedia, Porphyromonas gingivalis, Porphyromonas endodontalis, Peptostreptococcus micros, Enterococcus sp., Streptococcus sp., Fusobacterium nucleatum, Tannerella forsythensis and Treponema denticola. Since members of the domain Archaea have been implicated in the severity of periodontal disease, and a recent report confirms that archaea are present in endodontic infections, $16 \mathrm{~S}$ archaeal primers were also used to detect which patients carried these prokaryotes, to determine if their presence correlated with severity of the clinical symptoms. A Methanobrevibacter oralis-like species was detected in one asymptomatic and one symptomatic patient. DNA from root canals of these two patients was further analysed using species-specific primers to determine bacterial cohabitants. Trep. denticola was detected in the asymptomatic but not the symptomatic patient. Conversely, Porph. endodontalis was found in the symptomatic but not the asymptomatic patient. All other species except enterococci were detected with the species-specific primers in both patients. These results confirm the presence of archaea in root canals and provide additional insights into the polymicrobial communities in endodontic infections associated with clinical symptoms.
\end{abstract}

\section{INTRODUCTION}

The use of molecular mechanisms for defining the taxonomy of the oral microbial flora has emphasized the complexity of these communities and has confirmed estimates that more than 500 bacterial species exist in the oral cavity (Paster et al., 2001). The composition of these complex microbial communities varies in different ecological niches, such as the gingival crevice, tongue, buccal mucosa and saliva. Less attention has been paid to the

Abbreviation: RDP-II, Ribosomal Database Project II.

The GenBank/EMBL/DDBJ Trace Archive accession numbers for the bacterial 16S rDNA sequences are 1391568375-1391571307, and for the archaeal 16S rDNA sequences 1391568038-1391568374 and 1391557217-1391557263.

A table showing the taxonomic distribution of bacterial phylotypes detected in the study is available as supplementary data with the online version of this paper. microbial flora in infected root canals of teeth. Bacteria can gain access to the dental pulp through the crown or root surfaces in association with processes such as caries, periodontal disease or trauma. Any micro-organisms colonizing the normally sterile root-canal space can cause pulp necrosis and inflammation in the surrounding bone. The microbial community within the root canal is thought to undergo ecological succession as nutritional, acidic and oxygenation changes occur in conjunction with bacterial growth (Sundqvist \& Figdor, 2003). This chronic infectious process can progress asymptomatically, but dramatic clinical symptoms such as pain and swelling can occur. Although the patient's immune system plays a key role in the response to endodontic infections, qualitative and quantitative changes in the bacterial composition within the root canal have been implicated in clinical presentation. Although bacterial cultivation studies have suggested that specific bacterial species such as strict anaerobes are associated with symptoms (Jacinto et al., 2003; Drucker \& 
Natsiou, 2000), these analyses have not been definitive (Sakamoto et al., 2006; Siqueira \& Rocas, 2005).

The development of 16S rRNA phylogenetic methodology has widened the scope of detectable micro-organisms to include uncultivable organisms that may play significant, as yet undefined, roles in pathogenesis (Eckburg et al., 2003; Kroes et al., 1999; Paster et al., 2001). This methodology has been applied to characterization of oral microbial populations of healthy patients (Aas et al., 2005), patients with periodontal disease (Kroes et al., 1999), and patients with endodontic infections (Munson et al., 2002; Saito et al., 2006; Sakamoto et al., 2006). The latter studies indicate that the root-canal flora contains novel phylotypes and is more complex than culturing studies have previously indicated. Furthermore, recent studies have suggested that members of the domain Archaea, prokaryotes that have been implicated as pathogens in human disease (Eckburg et al., 2003), are found in greater abundance in dental plaque from sites with periodontal disease than in plaque from non-diseased sites (Lepp et al., 2004). Although attempts to isolate archaea from root-canal samples by amplification of $16 \mathrm{~S}$ rDNA were at first unsuccessful (Siqueira et al., 2005), one recent study has confirmed that members of the methanogenic archaea, similar to Methanobrevibacter oralis, can be detected in rootcanal samples (Vianna et al., 2006). The purpose of this study was twofold: first, to use PCR amplification of $16 \mathrm{~S}$ rRNA sequences to identify bacterial phyla present in a pool of infected teeth of patients with and without clinical symptoms; and second, to confirm the presence of archaea in infected root canals and determine if their presence correlated with disease severity. In the present study, $M$. oralis-like $16 \mathrm{~S}$ rDNA was amplified from one symptomatic and one asymptomatic patient. Root-canal DNA of these two patients was further analysed for bacterial content using species-specific primers.

\section{METHODS}

Patient selection. Patients over the age of 21 years who presented to the University of Buffalo Graduate Endodontics Clinic for treatment were assessed. Data collected for each patient consisted of gender, clinical examination results, diagnosis, duration of symptoms associated with the dental condition (e.g. pain, swelling), and any concomitant drug therapy and medical conditions. In accordance with University at Buffalo Human Institutional Review Board requirements, the patients in this review board-approved study were de-identified so that the collected history and specimen for each patient were correlated only by randomly assigned numbers.

Criteria for inclusion. Healthy patients with teeth that had no previous pulpal treatment and who were diagnosed with pulp necrosis, based on negative responses to thermal stimulation and electronic pulp-vitality testing when possible, and with chronic apical periodontitis, based on radiographic evidence of periapical radiolucency, were included in the study. Single and multirooted teeth were included. Patients with a history of antibiotic treatment within the previous 3-month period were excluded. Subjects were enrolled regardless of race, and attempts were made to include equivalent numbers of male and female patients. Female subjects were excluded on the basis of pregnancy or lactation. Only one tooth was sampled from each patient.

Patient classification. Patients were classified as symptomatic if they had an immediate history of spontaneous pain, pain to percussion, or pain upon palpation. The presence of swelling, lymphadenopathy or evidence of a sinus tract was considered symptomatic whether or not pain was present. Patients without the above criteria were considered asymptomatic. Of the 34 patients who met the criteria for inclusion in the study, 20 patients, including nine males and 11 females, were classified as symptomatic, and 14 patients, including eight males and six females, were classified as asymptomatic.

Endodontic sample collection. Samples from infected root canals were collected using a modification of published methods (Fouad et al., 2002). The tooth was isolated with a dental dam and the surrounding field was cleaned with $30 \%$ hydrogen peroxide and decontaminated with $2.5 \%$ sodium hypochlorite for $30 \mathrm{~s}$. After removal of caries, involved dentin and existing restorations, the area was swabbed with $2.5 \%$ sodium hypochlorite, then deactivated with sterile $5 \%(\mathrm{w} / \mathrm{v})$ sodium thiosulfate. Access to the pulp chamber and root canal was made with a new, sterile, \#4 round bur without water spray. After minimal canal enlargement with sterile saline irrigant to allow access to the working length, dry, autoclaved paper points were placed within the canal space for 30 seconds. The paper points containing the absorbed root-canal contents were placed in sterile vials containing $1 \mathrm{ml} 10 \mathrm{mM}$ Tris- $\mathrm{HCl}, 1 \mathrm{mM}$ EDTA, $\mathrm{pH} 8$. Samples were then taken directly to the laboratory and stored at $-80^{\circ} \mathrm{C}$ until processing. Endodontic treatment proceeded routinely.

Preparation of microbial DNA from root-canal contents. Each sample was thawed on ice, vortexed for $2 \mathrm{~min}$ and centrifuged at $2000 \mathrm{~g}$ for $5 \mathrm{~min}$. After removal of the supernatant and paper point, the pellet, containing microbial cells, was resuspended in a solution containing $100 \mu \mathrm{l}$ sterile molecular biology grade water, $100 \mu \mathrm{l} 0.1 \%$ $(\mathrm{w} / \mathrm{v})$ blue dextran solution, $200 \mu \mathrm{l}$ cell lysis buffer $(100 \mathrm{mM}$ Tris$\mathrm{HCl}, \mathrm{pH}$ 7.4, $20 \mathrm{mM}$ EDTA, $5 \mathrm{M}$ guanidine thiocyanate, $2 \%$ Triton $\mathrm{X}-100)$ and $100 \mu \mathrm{g}$ proteinase $\mathrm{K}$, and incubated at $65^{\circ} \mathrm{C}$ for $30 \mathrm{~min}$. This mixture was then added to a tube containing $350 \mu \mathrm{g}$ of sterile glass beads (FastRNA Pro Blue kit, Qbiogene) and placed in a FastPrep cell disrupter (Qbiogene) at $4 \mathrm{~m} \mathrm{~s}^{-1}$ for $30 \mathrm{~s}$. A $400 \mu \mathrm{l}$ volume of $100 \%$ benzyl alcohol was added and the solution was vortexed for $15 \mathrm{~s}$. Nucleic acids were separated from cell-lysis products by centrifugation at $7000 \mathrm{~g}$ for $5 \mathrm{~min}$. The upper aqueous phase was transferred to a new tube and precipitated with

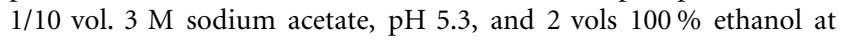
$-20{ }^{\circ} \mathrm{C}$. The solution was then centrifuged at $16000 \mathrm{~g}$ for $30 \mathrm{~min}$ to pellet the nucleic acids, which were then RNase-treated, reprecipitated and resuspended in $30 \mu \mathrm{l} 10 \mathrm{mM}$ Tris- $\mathrm{HCl}, 1 \mathrm{mM}$ EDTA, $\mathrm{pH}$ 8. Aliquots were stored at $-80^{\circ} \mathrm{C}$ until used as template in PCR. The mean amount of DNA recovered per tooth was $8.8 \pm 3.8 \mu \mathrm{g}$, as determined spectrophotometrically. The recovered root-canal contents contained both human host and microbial DNA, so microbial target template concentrations could not be readily quantified. To examine the overall composition of the microbial communities in the asymptomatic and symptomatic groups, equal volumes of DNA from each patient preparation were pooled according to the asymptomatic and symptomatic classification.

Universal bacterial primers and PCR conditions. Each PCR mix contained $\sim 250 \mathrm{ng}$ DNA, $100 \mathrm{ng}$ each forward and reverse primer, $200 \mathrm{mM}$ dNTPs (Invitrogen), $25 \mathrm{mM} \mathrm{MgCl}_{2}$ and $2.5 \mathrm{U}$ Platinum Taq DNA polymerase (Invitrogen) in the manufacturer's buffer. All reactions were done with a $3 \mathrm{~min} 95^{\circ} \mathrm{C}$ hot start followed by 35 cycles of $95^{\circ} \mathrm{C}$ for $1 \mathrm{~min}$, the primer-specific annealing temperature for $1 \mathrm{~min}$, and $72{ }^{\circ} \mathrm{C}$ for $2 \mathrm{~min}$, followed by a $30 \mathrm{~min} 72^{\circ} \mathrm{C}$ 
extension. Primers designed to anneal to conserved regions were used to amplify bacterial $16 \mathrm{~S}$ rDNA sequences that contained intervening species-specific regions. The forward primer, 8F (5'-AGAGTTTGATCCTGGCTCAG-3'), was used with reverse primers $1391 \mathrm{R}$ $\left(5^{\prime}\right.$-GACGGGCGGTGTGTRCA-3') or 1492R (5'-GGGTTACCTTGTTACGACTT-3') with an annealing temperature of $56^{\circ} \mathrm{C}$ and template DNA from the root-canal contents to amplify 1.4 and $1.5 \mathrm{kbp}$ regions, respectively, of bacterial 16S rDNA (Baker et al., 2003). The products of primers $8 \mathrm{~F}$ and $1391 \mathrm{R}$ and the products of primers $8 \mathrm{~F}$ and $1492 \mathrm{R}$ with pooled DNA from asymptomatic patients as template, and the products of the same primer pairs using DNA template pooled from symptomatic patients, were cloned for sequence analysis. An equivalent number of clones were analysed from both the symptomatic and asymptomatic patients, for a total of $\sim 1500$ clones.

Detection of archaeal and bacterial species with specific oligonucleotide primers. Amplicon products of bacterial universal primers were used as templates for detection of Prevotella intermedia, Porphyromonas endodontalis, Porphyromonas gingivalis, Peptostreptococcus micros, Streptococcus sp., Fusobacterium nucleatum and Tannerella forsythensis using species-specific primers and annealing temperatures as shown in Table 1. Representative strains of each bacterial species were used as positive controls to optimize PCR conditions for each primer pair; limits of detection were determined with serial dilutions of DNA prepared from pure cultures using standard alkaline-lysis techniques (Sambrook et al., 1989). Unamplified DNA pools from asymptomatic and symptomatic patients were used directly with primers to amplify the $16 \mathrm{~S} \mathrm{rDNA}$ of archaea and Treponema denticola, which would not be amplified well with the bacterial universal primers (Choi et al., 1994; Kroes et al., 1999), and to amplify the tuf gene, encoding the Tu elongation factor, of Enterococcus sp. (Table 1). Individual patient samples were screened for the presence of archaea to determine which patients in the pools carried these micro-organisms. DNA samples from archaea-positive patients were individually characterized with bacterial species-specific primers. All negative reactions were repeated at least twice with unamplified DNA template to confirm that the target sequences were below detectable threshold levels. To confirm the specificity of the species-specific primers, randomly chosen cloned amplicons from each positive reaction were sequenced and confirmed to have $\geqslant 99 \%$ similarity to GenBank entries for each species.

Cloning and sequencing. The completed PCR mixtures were electrophoresed on agarose gels; amplicons were eluted from the gel with a Qiaex bead kit (Qiagen) and cloned into pCR-4-TOPO vectors (Invitrogen). One-Shot TOP10 Competent Escherichia coli cells (Invitrogen) were transformed using the TOPO-TA Cloning kit (Invitrogen) according to the manufacturer's instructions. Plasmid DNA from each transformant was prepared by a modified alkalinelysis method. Nucleotide sequences of the clone inserts were determined by cycle sequencing using BigDye Terminator (Applied Biosystems) and 3.2 pmol M13F (5'-GTAAAACGACGGCCAG-3') and M13R (5'-CAGGAAACAGCTATGAC-3') sequencing primers. Sequences were analysed on ABI 3730xl sequencers (Applied Biosystems). After trimming to remove vector sequence and adjusting for quality values, the mean single-sequence read length was $\sim 700$ nt. Bidirectional sequence reads of clone inserts provided near full-length $16 \mathrm{~S}$ bacterial and archaeal sequences, which were used for phylogenetic analysis.

Phylogenetic analysis. To identify bacterial rDNA amplified with the universal primers, sequences with $\geq 97 \%$ similarity were assembled and the individual assemblies were analysed by comparison with known phylotypes via the Sequence Match program of the Ribosomal Database Project II (RDP-II; version 3 release 9.38) (Cole et al., 2005). The relationships of the cloned assemblies to known

Table 1. PCR primers and conditions for microbial detection

Bold type in the primer sequence or annealing temperature indicates a modification from the reference listed.

\begin{tabular}{|c|c|c|c|c|}
\hline Micro-organism & Forward and reverse primers & $\begin{array}{c}\text { Annealing } \\
\text { temperature }\left({ }^{\circ} \mathrm{C}\right)\end{array}$ & $\begin{array}{l}\text { Amplicon } \\
\text { size (bp) }\end{array}$ & Reference \\
\hline Prev. intermedia & $\begin{array}{l}\text { 5'-CGTGGACCAAAGATTCATCGGTGGA-3' } \\
5^{\prime} \text {-CCGCTTTACTCCCCAACAAA-3' }\end{array}$ & 57 & 259 & Baumgartner et al. (1999) \\
\hline Porph. endodontalis & $\begin{array}{l}\text { 5'-GCTGCAGCTCAACTGTAGTC-3' } \\
5^{\prime} \text {-CCGCTTCATGTCACCATGTC-3' }\end{array}$ & 57 & 665 & Bogen \& Slots (1999) \\
\hline Porph. gingivalis & $\begin{array}{l}\text { 5'-AGGCAGCTTGCCATACTGCG-3' } \\
\text { 5'-ACTGTTAGCAACTACCGATGT-3' }\end{array}$ & 57 & 404 & Bogen \& Slots (1999) \\
\hline Pept. micros & $\begin{array}{l}\text { 5'-AGAGTTTGATCCTGGCTCAG-3' } \\
\text { 5'-ATATCATGCGATTCTGTGGTCTC-3' }\end{array}$ & 57 & 207 & Conrads et al. (1997b) \\
\hline Streptococcus sp. & $\begin{array}{l}\text { 5'-AGAGTTTGATCCTGGCTCAG-3' } \\
\text { 5'-GTACCGTCACAGTATGAACTTTCC-3' }\end{array}$ & 55 & 500 & Conrads et al. (1997a) \\
\hline Fusob. nucleatum & $\begin{array}{l}\text { 5'-AGAGTTTGATCCTGGCTCAG-3' } \\
\text { 5'-GTCATCGTGCACACAGAATTGCTG-3' }\end{array}$ & 55 & 407 & Conrads et al. (1997a) \\
\hline Tann. forsythensis & $\begin{array}{l}\text { 5'-TACAGGGGAATAAAATGAGATACG-3' } \\
5^{\prime} \text {-ACGTCATCCCAACCTTCCTC-3' }\end{array}$ & 57 & 745 & Tran \& Rudney (1999) \\
\hline Trep. denticola & $\begin{array}{l}\text { 5'-TAATACCGAATGTGCTCATTTACAT-3' } \\
5^{\prime} \text {-TCAAAGAAGCATTCCCTCTTCTTCTTA-3' }\end{array}$ & 60 & 316 & Ashimoto et al. (1996) \\
\hline Archaea & $\begin{array}{l}\text { 5'-TCCAGGCCCTACGGG-3' - } \\
5^{\prime}-\text { YCCGGCGTTGAMTCCAATT-3' }\end{array}$ & 50 & 607 & Lepp et al. (2004) \\
\hline Enterococcus sp. & $\begin{array}{l}\text { 5'-TACTGACAAACCATTCATGATG-3' } \\
5^{\prime} \text {-AACTTCGTCACCAACGCGAAC-3' }\end{array}$ & 55 & 112 & Ke et al. (1999) \\
\hline
\end{tabular}


phylotypes were expressed with S_ab scores, defined as the number of unique oligomers shared between the query sequence and the sequence in the RDP-II database, divided by the lowest number of unique oligomers in either of the two sequences. The top 20 hierarchical matches for each assembly consensus sequence were examined, and the sequence was designated the phylotype in the RDP-II database with the highest S_ab score. In the two cases in which the assembly sequences matched more than one phylotype with the same S_ab score, data are presented as the type species. Phylogenetic identification was verified to the genus level using the RDP-II database Classifier program, which uses a naive Bayesian rRNA classifier (version 1.0) to assign a sequence to a taxonomical hierarchy according to Holt et al. (1994).

\section{RESULTS AND DISCUSSION}

\section{Sequence analysis of cloned 16S rDNA amplicons from universal primers}

Cloned amplicons generated from PCR with DNA from the two patient pools and universal primers were sequenced, assembled and compared with sequences of known species in the RDP-II database for phylotype identification. In some cases, multiple different assemblies matched most closely to the same sequence in the RDP-II database, but with a different similarity, as indicated by the Sequence Match (S_ab) score. Sequences were confirmed to the genus level with a greater than $95 \%$ confidence threshold for symptomatic and asymptomatic samples, using the RDP-II database Classifier program. Taxonomic classifications and distribution of most prevalent phylotypes are shown in Supplementary Table S1 in JMM online. Asymptomatic and symptomatic patients had only seven phylotypes in common: Dialister invisus (GenBank accession no. AF287787); Fusob. nucleatum [most similar to phylotype ChDC OS50 (AF543300)]; Prevotella sp. [most similar to oral clone F045 (AY005056)]; Veillonella parvula (AY995769), Lactobacillus gasseri (AY730721), Solobacterium moorei (AY044915) and Atopobium parvulum (X67150). The remaining clones were exclusive to either the asymptomatic or the symptomatic patients, although multiple assemblies matched most species (Table 2). The results of this study support recent studies using culture-independent methods that indicate that the polymicrobial communities in infected root canals may be more complex than previously appreciated (Sundqvist \& Figdor, 2003; Siqueira \& Rocas, 2005). A recent review (Siqueira \& Rocas, 2005) of studies that use molecular methodologies has indicated that independently of the presence of symptoms, the most prevalent non-treponemal endodontic bacteria are Porph. endodontalis, Tann. forsythensis, Pseudoramibacter alactolyticus, Dialister pneumosintes, Filifator alocis, Porph. gingivalis and Propionibacterium propionicum. In the present study, universal primers did not detect Porph. endodontalis, Tann. forsythensis or D. pneumosintes (Table 2), suggesting that they were not present in high abundance in our patient pools or that the universal primers did not anneal well to the $16 \mathrm{~S}$ rDNA of these species.

\section{Comparison of amplicons from universal and species-specific primers}

Although the universal primers detected a wide range of bacterial phylotypes, PCR with species-specific primers confirmed the limitations of universal primers, which cannot anneal equally well to all microbial $16 \mathrm{~S}$ rDNA (Baker et al., 2003; de Lillo et al., 2006) and did not amplify DNA from all the bacteria present in the template. Similarly, the number of PCR cycles could bias amplification in favour of more abundant species by allowing self-annealing in later amplification cycles (Acinas et al., 2005), resulting in a less representative sample of the community. Although Prev. intermedia was not detected with universal primers in either asymptomatic or symptomatic patients (Table 2), speciesspecific primers were able to successfully amplify Prev. intermedia $16 \mathrm{~S}$ rDNA with $\geq 99 \%$ similarity to the expected sequence from a pool of DNA from symptomatic patients (Table 3). However, species-specific primers could not detect Prev. intermedia in the DNA of asymptomatic patients, suggesting that the relative numbers of this species may be lower in these patients. Conversely, universal primers detected peptostreptococci only in the asymptomatic patients (Table 2), whereas the species-specific primers were able to detect Pept. micros in both patient groups (Table 3). Similarly, Porph. endodontalis and Tann. forsythensis, which were not detected with universal primers (Table 2), were successfully amplified from both asymptomatic and symptomatic patients when species-specific primers were used (Table 3). Streptococcus sp. and Fusob. nucleatum were detected with both universal and speciesspecific primers (Tables 2 and 3 ) from asymptomatic and symptomatic patients. Although Porph. gingivalis was detected with universal primers only in asymptomatic patients (Table 2), species-specific primers detected this species in both patient groups (Table 3). As expected, Trep. denticola was detected only with species-specific primers. However, this species was detected only in the asymptomatic patients (Table 3), suggesting that it is absent or less abundant in the symptomatic patients, in contrast to reports elsewhere (Siqueira \& Rocas, 2005). Enterococcal speciesspecific primers did not amplify the tuf gene from patient DNA, even when the amount of template in the reaction mix was increased by up to threefold. The absence of an enterococcal tuf amplicon was supported by the lack of $16 \mathrm{~S}$ rDNA universal primer amplicons that were $\geq 60 \%$ similar to any enterococcal species in the RDP-II database (data not shown). Nevertheless, other studies have also failed to detect this species in root canals (Cheung \& Ho, 2001), and the abundance of Enterococcus faecalis in root canals has been reported to be variable (Sedgley et al., 2006).

\section{Bacterial communities in asymptomatic and symptomatic patients}

The bacterial phyla detected with universal primers included Actinobacteria, Bacteroidetes, Firmicutes, Fusobacteria and Proteobacteria (Supplementary Table S1 in JMM online). Although others have also detected low levels of 
Table 2. Bacterial species and phylotypes detected in each group of patients by amplification of $16 \mathrm{~S}$ rDNA with universal primers

\begin{tabular}{|c|c|c|c|}
\hline Asymptomatic ${ }^{\star}$ & S_ab range $\dagger$ & Symptomatic & S_ab range \\
\hline Anaeroglobus geminatus & $0.644-0.757$ & Eubacterium sp. oral clone BU061 & 0.985 \\
\hline Bacteroidales oral clone MCE7_20 & $0.708-0.986$ & Fusob. nucleatum & $0.714-0.990$ \\
\hline Brachymonas denitrificans & 0.835 & Fusobacterium sp. oral clone CY024 & 0.786 \\
\hline Campylobacter gracilis & $0.744-0.985$ & Fusobacterium sp. oral clone CZ006 & 0.975 \\
\hline Catonella morbi & $0.754-0.980$ & Granulicatella adiacens & 0.986 \\
\hline Cryptobacterium curtum & 0.909 & Lactobacillus johnsonii & 0.770 \\
\hline Desulfovibrio sp. D4 & 0.993 & Lactobacillus paracasei & 0.845 \\
\hline Desulfovibrio sp. LNB1 & 0.721 & Lactobacillus salivarius & $0.929-1.000$ \\
\hline Dialister (genus), uncultured bacterium & $0.656-0.951$ & Mogibacterium neglectum & 0.983 \\
\hline Dialister sp. E2_20 & $0.853-0.975$ & Neisseria bacilliformis & $0.950-0.959$ \\
\hline Eikenella corrodens & 0.917 & Neisseria flava & $0.972-0.981$ \\
\hline Eikenella sp. MDA2346-4 & 0.958 & Prevotella melaninogenica & 0.958 \\
\hline Eubacterium (genus), Eubacteriaceae oral clone P2PB_46 P3 & $0.808-0.957$ & Prevotella nigrescens & 0.992 \\
\hline Eubacterium infirmum & $0.776-0.927$ & Prevotella sp. oral clone FU048 & $0.928-0.980$ \\
\hline Eubacterium nodatum & 0.961 & Rothia dentocariosa & 0.994 \\
\hline Eubacterium sp. oral clone JS001 & 0.764 & Sphingomonas sp. P2 & 0.990 \\
\hline Eubacterium sp. oral strain A35MT & $0.609-0.979$ & Streptococcus gordonii & $0.932-0.985$ \\
\hline Eubacterium sulci & 0.936 & Streptococcus mitis & $0.695-0.988$ \\
\hline Filif. alocis & $0.751-0.959$ & Streptococcus mutans & 0.917 \\
\hline Firmicutes oral clone CK057 & 0.821 & Streptococcus oralis & $0.744-0.988$ \\
\hline Firmicutes oral clone MCE3_120 & $0.647-0.901$ & Streptococcus parasanguinis & $0.797-0.991$ \\
\hline Firmicutes sp. oral strain FTB41 & 0.740 & Streptococcus salivarius & 1.000 \\
\hline Fusobacterium genomosp. C2 & 0.933 & Streptococcus sanguinis & $0.979-0.984$ \\
\hline Fusob. nucleatum subsp. animalis & 0.959 & Veillonella sp. oral clone AA050 & 0.811 \\
\hline Haemophilus aphrophilus & $0.935-0.975$ & Veillonella sp. oral clone X042 & $0.948-0.965$ \\
\hline Haemophilus paraphrophilus & 0.979 & & \\
\hline Lactobacillus catenaformis & 0.957 & & \\
\hline Lactobacillus plantarum & 0.835 & & \\
\hline Megasphaera sp. oral clone MCE3_141 & 0.970 & & \\
\hline Mogibacterium timidum & $0.710-0.953$ & & \\
\hline Olsenella profusa & $0.953-0.981$ & & \\
\hline O. uli & $0.660-0.975$ & & \\
\hline Parascardovia denticolens & 0.984 & & \\
\hline Pept. micros & $0.742-1.000$ & & \\
\hline Peptostreptococcus sp. oral clone BS044 & $0.741-0.969$ & & \\
\hline Peptostreptococcus stomatis & 0.743 & & \\
\hline Peptostreptococcus sp. oral clone FG014 & $0.721-0.830$ & & \\
\hline Peptostreptococcus sp. oral clone FL008 & $0.721-0.975$ & & \\
\hline Porph. gingivalis & 0.998 & & \\
\hline Porphyromonas sp. oral clone P2PB_52 P1 & $0.717-0.968$ & & \\
\hline Prevotella baroniae & $0.764-0.980$ & & \\
\hline Prevotella buccae & $0.773-0.961$ & & \\
\hline Prevotella denticola & 0.961 & & \\
\hline Prevotella genomosp. C2 & $0.910-0.948$ & & \\
\hline Prevotella genomosp. P4 & $0.822-0.978$ & & \\
\hline Prevotella oralis & 0.964 & & \\
\hline Prevotella oulorum & 0.958 & & \\
\hline Prevotella sp. E7_34 & $0.675-0.989$ & & \\
\hline Prevotella sp. oral clone DA058 & 0.967 & & \\
\hline Prevotella tannerae & $0.877-0.960$ & & \\
\hline Propionibacterium avidum & 0.978 & & \\
\hline Prop. propionicum & 0.837 & & \\
\hline Pseud. alactolyticus & $0.734-0.994$ & & \\
\hline
\end{tabular}


Table 2. cont.

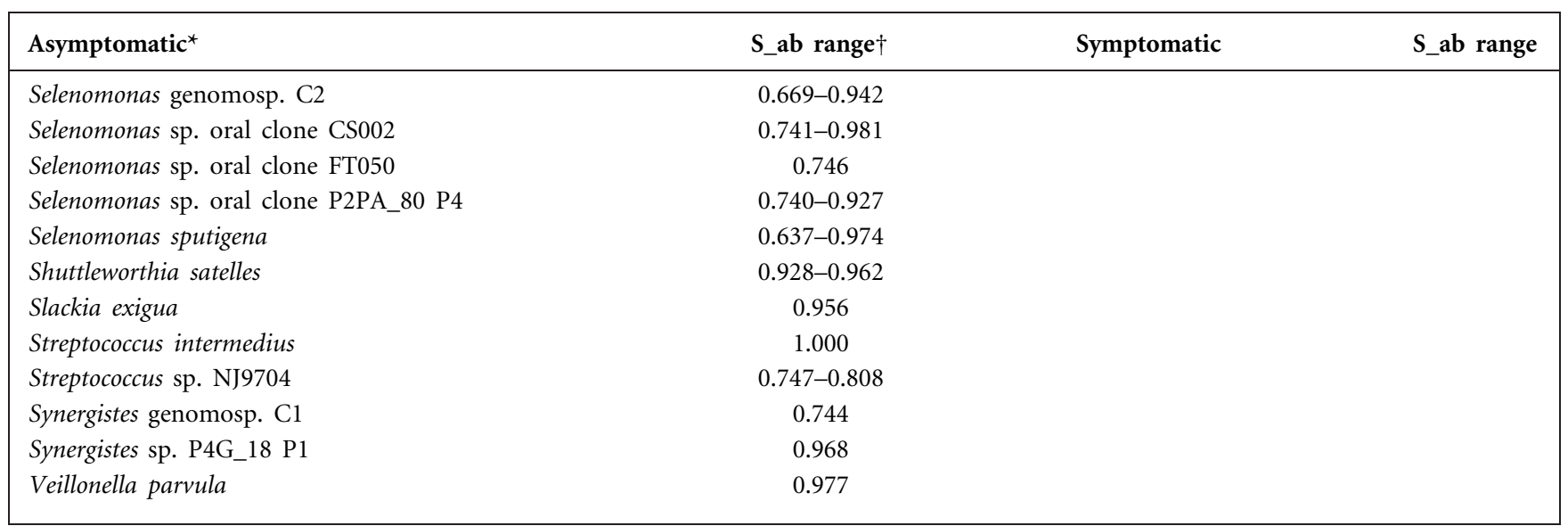

*The PCR template was a pool of equal volumes of DNA prepared from each of the asymptomatic patients $(n=14)$ or symptomatic patients $(n=20)$. Each species listed is the reference strain in the RDP-II database that had the highest similarity to the queried assembly sequences using the Sequence Match program. Entries in bold type indicate that members of these genera were unique to the symptomatic patient pool. $\dagger S \_a b$ scores indicate the degree of match of assembly consensus sequences to each named phylotype in the RDP-II database. The top 20 hits were examined. In cases where the assemblies matched more than one phylotype in the database with the same S_ab score, the ATCC type strain matched is shown. When multiple different assemblies matched the same phylotype, the range of S_ab scores is shown.

Deferribacteres (Saito et al., 2006), none was detected in the present study. Similarly, the TM7 phylotype, which has been reported in dental plaque (Brinig et al., 2003) and endodontic samples (de Lillo et al., 2006), was not detected. Bacterial species previously reported as potential pathogens in endodontic infections were detected. D. invisus, Olsenella uli and Synergistes sp., which have been recovered from symptomatic infections in earlier studies (Rocas \& Siqueira, 2005), were detected in our asymptomatic, but not symptomatic patients (Table 2). Granulicatella adiacens, which has been reported in symptomatic patients (Rocas \& Siqueira, 2005), was found exclusively in symptomatic patients (Table 2), supporting the possibility that this species has traits associated with clinical symptoms, or

Table 3. Microbial amplicons detected in root-canal samples with universal archaeal and species-specific primers

\begin{tabular}{|c|c|c|c|c|}
\hline Micro-organism detected & Asymptomatic pooled ${ }^{\star}$ & Symptomatic pooled & Asymptomatic patient \#10 & Symptomatic patient \#37 \\
\hline Prev. intermedia & $-\ddagger$ & + & - & + \\
\hline Porph. gingivalis & + & + & + & + \\
\hline Pept. micros & + & + & + & + \\
\hline Tann. forsythensis & + & + & + & + \\
\hline Trep. denticola & + & $-\|$ & + & - \\
\hline Enterococcus sp. & -9 & - & - & - \\
\hline
\end{tabular}

${ }^{\star}$ Template DNA was a pool of equal volumes either from each of the asymptomatic patients $(n=14)$ or from each of the symptomatic patients $(n=20)$. DNA preparations from root canals of patients $\# 10$ and $\# 37$, the only patients with detectable archaea, were further examined individually.

$\dagger+$, a detectable PCR product that was confirmed by nucleotide sequence analysis to be $\geqslant 99 \%$ similar to the expected species sequence; - , no amplicon detected when entire reaction was electrophoresed on $0.7 \%$ agarose gel and stained with ethidium bromide.

$\ddagger \geqslant 0.1 \mathrm{ng}$ Prev. intermedia ATCC 25611 chromosomal DNA was necessary for detection of this amplicon under the reaction conditions in this study.

$\S \geqslant 0.05$ ng Porph. endodontalis ATCC 35406 chromosomal DNA was necessary for detection of this amplicon.

$\| \geqslant 0.04 \mathrm{ng}$ Trep. denticola ATCC 35405 chromosomal template was necessary for detection of this amplicon.

$\mathbf{S} \geqslant 0.05$ ng Ent. faecalis strain $\mathrm{JH} 2-2$ chromosomal DNA was necessary for detection of this amplicon. 
that it thrives in multispecies communities associated with symptoms. The prevalence of amplicons that were most similar to Fusob. nucleatum in the symptomatic patients was noteworthy (Supplementary Table S1), supporting previous studies with broad-range PCR primers that suggest that levels of this species may be greatly underestimated in endodontic abscesses (Wade et al., 1997). The symptomatic patients also had a greater number of facultative streptococci than the asymptomatic patients, suggesting that the former infections may have a less reduced microbial community, consistent with earlier infections with a higher $\mathrm{pO}_{2}$ (Sundqvist \& Figdor, 2003). Overall, the diversity of the microflora of asymptomatic patients was more complex, with a larger number of phylotypes represented (Table 2). The approach of pooling DNA from patients in the asymptomatic and symptomatic groups and sequencing a large number of clones was aimed at minimizing the bias of patient variability. Nevertheless, the point at which an infection progresses to cause clinical symptoms may be affected by host variables in addition to the bacterial composition of the infection. Indeed, relative abundance rather than the presence of specific bacteria may also affect symptoms (Drucker \& Natsiou, 2000). Quantification was not a goal of the present study, but future analyses using methodologies such as quantitative real-time PCR may clarify this issue.

\section{Clinical presentations of patients carrying archaea in root canals}

Universal primers for archaeal 16S rDNA amplified a $\sim 600$ bp product from both pools of DNA from asymptomatic and symptomatic patients. In order to identify which patients had archaea in their root-canal infections, the DNA from each individual patient in the pools was used as template in PCR. Amplicons were recovered from two patients. The first, designated patient \#10, was female and classified as asymptomatic. Her upper-left second premolar had recurrent decay around a large amalgam restoration and a $2 \mathrm{~mm}$ apical radiolucency. The patient reported no previous or current symptoms. The tooth tested non-vital with no response to electrical pulp testing or thermal stimuli. There was no sensitivity to palpation or percussion. The associated medical history indicated that the patient was taking birth-control pills, iron supplements and albuterol for asthma.

The second patient, designated patient \#37, was male and classified as symptomatic. He presented with a 1-month history of spontaneous, intermittent, throbbing pain that was elicited by pressure and mastication, and that was relieved with non-steroidal anti-inflammatory agents. Clinical examination showed a large composite restoration with recurrent marginal decay on his upper-left first premolar. The tooth gave no response to stimulation with cold or electric pulp testing, indicating pulp necrosis, and was sensitive to palpation and percussion. Although there was no intraoral or extraoral swelling, there was a sinus tract exiting to the buccal mucosa that was traced to the $4 \times 5 \mathrm{~mm}$ apical radiolucency associated with the infected premolar. The patient had a history of hypertension satisfactorily controlled with beta-blockers. The patient reported no other medical conditions, and had taken no other medications except for ibuprofen for toothache pain during the previous 3 -month period. In both patient \#10 and patient \#37, the overall surrounding periodontal conditions were sound. Adjacent teeth were healthy, responding normally to vitality tests, with no pocketing $\geq 3 \mathrm{~mm}$.

\section{Detection of $\boldsymbol{M}$. oralis-like clones}

Archaeal amplicons from the pools and from each patient were cloned. Up to six randomly selected clones of each of the pooled or individual patient templates were sequenced. Although these amplicons theoretically contained $16 \mathrm{~S}$ rDNA sequences from all the archaeal phylotypes present in the root canals, all of the cloned sequences agreed with each other, suggesting that the amplified sequence represented either the only, or the predominant, member of this species. In all instances, the sequences of the archaeal amplicons were $100 \%$ identical to that of an uncultured M. oralis-like species first isolated from the oral cavity (GenBank accession no. AY374553; Lepp et al., 2004). The sequences were also $100 \%$ identical to one of the two Methanobrevibacter species identified in root-canal contents by $16 \mathrm{~S}$ rDNA amplification (GenBank accession no. DQ251044; Vianna et al., 2006).

As with our results comparing species-specific and universal primers, the detection of archaea in root-canal samples emphasizes the limits of detection and sensitivity of PCR methodologies. Stringencies of PCR conditions (e.g. $\mathrm{Mg}^{2+}$ concentrations, temperatures and times) and primer sequences can result in over- or underdetection of target DNA that may, in turn, lead to incorrect conclusions about the presence or absence of a microbial species. Previous studies using different oligonucleotide primers have been unsuccessful at amplifying archaeal DNA (Siqueira et al., 2005). However, with the primers and conditions used in our study, archaeal 16S rDNA amplicons were readily detected. Our PCR conditions differed from those used to identify the same species of archaea in asymptomatic patients with root-canal infections (Vianna et al., 2006). Although the presence of archaea in subgingival plaque has been correlated with the severity of periodontal disease (Lepp et al., 2004), the present study detected archaea in both asymptomatic and symptomatic patients. Patient \#37 had a draining sinus tract that may have allowed ingress of oral bacteria. No such obvious route of entry was clinically obvious in the asymptomatic patient \#10. Furthermore, both patients had healthy surrounding periodontium. The medical and dental histories may provide future insights into the role that archaea and other microbial species play in the disease process in individual patients.

\section{Archaeal-bacterial communities}

The archaeon genus detected in these studies, Methanobrevibacter, consists of strict anaerobes that 
metabolize hydrogen to produce methane. Although only one sequence of $M$. oralis-like amplicon was detected from the two individual patients, the sequence was identical to that of a clone previously identified from dental plaque and from root-canal contents. This low diversity of Methanobrevibacter 16S rDNA sequences isolated from different patients has been previously noted in oral and nonoral strains (Kulik et al., 2001). Root-canal DNA from patients \#10 and \#37 was examined with species-specific bacterial 16S rDNA primers (Table 3) to gain further insights into polymicrobial communities that contain Methanobrevibacter species. It has been suggested that metabolic competition for hydrogen with sulfate-reducing bacteria (Vianna et al., 2006), such as the Desulfovibrio species detected in the pooled DNA of asymptomatic patients in the present study, or treponemal species (Siqueira et al., 2005), might inhibit the coexistence of these bacteria with methanogenic archaea. Although the two patients with Methanobrevibacter were not examined for Desulfovibrio, they were examined for Trep. denticola. Interestingly, although patient \#37 supported this hypothesis and did not carry detectable levels of Trep. denticola, patient \#10 carried both the M. oralis-like strain and Trep. denticola. Future quantitative and qualitative studies of metabolic competitors in individual infected root canals colonized with methanogenic archaea may provide more insights into the nutritional interdependence of these microbes.

\section{ACKNOWLEDGEMENTS}

This work was supported by United States Public Health Service (USPHS) grants T32DE7034 and DE014868 and the University at Buffalo Division of Endodontics Funds. We thank Dr J. Cole of the RDP-II for his helpful advice. We also thank T. D. Felmet and A. R. Munsterman for excellent technical assistance, and members of the A. Sharma and J. Zambon laboratories (University at Buffalo Department of Oral Biology) who provided Prevotella, Porphyromonas, Fusobacterium and Tannerella strains used as positive controls in these studies.

\section{REFERENCES}

Aas, J. A., Paster, B. J., Stokes, L. N., Olsen, I. \& Dewhirst, F. E. (2005). Defining the normal bacterial flora of the oral cavity. J Clin Microbiol 43, 5721-5732.

Acinas, S. G., Sarma-Rupavtarm, R., Klepac-Ceraj, V. \& Polz, M. F. (2005). PCR-induced sequence artifacts and bias: insights from comparison of two 16S rRNA clone libraries constructed from the same sample. Appl Environ Microbiol 71, 8966-8969.

Ashimoto, A., Chen, C., Bakker, I. \& Slots, J. (1996). Polymerase chain reaction detection of 8 putative periodontal pathogens in subgingival plaque of gingivitis and advanced periodontitis lesions. Oral Microbiol Immunol 11, 266-273.

Baker, G. C., Smith, J. J. \& Cowan, D. A. (2003). Review and reanalysis of domain-specific $16 \mathrm{~S}$ primers. J Microbiol Methods 55, 541-555.
Baumgartner, J. C., Watkins, B. J., Bae, K. S. \& Xia, T. (1999). Association of black-pigmented bacteria with endodontic infections. J Endod 25, 413-415.

Bogen, G. \& Slots, J. (1999). Black-pigmented anaerobic rods in closed periapical lesions. Int Endod J 32, 204-210.

Brinig, M. M., Lepp, P. W., Ouverney, C. C., Armitage, G. C. \& Relman, D. A. (2003). Prevalence of bacteria of division TM7 in human subgingival plaque and their association with disease. Appl Environ Microbiol 69, 1687-1694.

Cheung, G. S. \& Ho, M. W. (2001). Microbial flora of root canaltreated teeth associated with asymptomatic periapical radiolucent lesions. Oral Microbiol Immunol 16, 332-337.

Choi, B. K., Paster, B. J., Dewhirst, F. E. \& Gobel, U. B. (1994). Diversity of cultivable and uncultivable oral spirochetes from a patient with severe destructive periodontitis. Infect Immun 62, 1889-1895.

Cole, J. R., Chai, B., Farris, R. J., Wang, Q., Kulam, S. A., McGarrell, D. M., Garrity, G. M. \& Tiedje, J. M. (2005). The Ribosomal Database Project (RDP-II): sequences and tools for high-throughput rRNA analysis. Nucleic Acids Res 33 (database issue), D294-D296.

Conrads, G., Gharbia, S. E., Gulabivala, K., Lampert, F. \& Shah, H. N. (1997a). The use of a 16S rDNA directed PCR for the detection of endodontopathogenic bacteria. J Endod 23, 433-438.

Conrads, G., Soffner, J., Pelz, K. \& Mutters, R. (1997b). Taxonomic update and clinical significance of species within the genus Peptostreptococcus. Clin Infect Dis 25 (suppl. 2), S94-S97.

de Lillo, A., Ashley, F. P., Palmer, R. M., Munson, M. A., Kyriacou, L., Weightman, A. J. \& Wade, W. G. (2006). Novel subgingival bacterial phylotypes detected using multiple universal polymerase chain reaction primer sets. Oral Microbiol Immunol 21, 61-68.

Drucker, D. B. \& Natsiou, I. (2000). Microbial ecology of the dental root canal. Microb Ecol Health Dis 12, 160-169.

Eckburg, P. B., Lepp, P. W. \& Relman, D. A. (2003). Archaea and their potential role in human disease. Infect Immun 71, 591-596.

Fouad, A. F., Barry, J., Caimano, M., Clawson, M., Zhu, Q., Carver, R., Hazlett, K. \& Radolf, J. D. (2002). PCR-based identification of bacteria associated with endodontic infections. J Clin Microbiol 40, 3223-3231.

Holt, J. G., Krieg, N. R., Sneath, P. H. A., Staley, J. T. \& Williams, S. T. (editors) (1994). Bergey's Manual of Determinative Bacteriology, 9th edn. Baltimore, MD: Williams \& Wilkins.

Jacinto, R. C., Gomes, B. P., Ferraz, C. C., Zaia, A. A. \& Filho, F. J. (2003). Microbiological analysis of infected root canals from symptomatic and asymptomatic teeth with periapical periodontitis and the antimicrobial susceptibility of some isolated anaerobic bacteria. Oral Microbiol Immunol 18, 285-292.

Ke, D., Picard, F. J., Martineau, F., Menard, C., Roy, P. H., Ouellette, M. \& Bergeron, M. G. (1999). Development of a PCR assay for rapid detection of enterococci. J Clin Microbiol 37, 3497-3503.

Kroes, I., Lepp, P. W. \& Relman, D. A. (1999). Bacterial diversity within the human subgingival crevice. Proc Natl Acad Sci U S A 96, 14547-14552.

Kulik, E. M., Sandmeier, H., Hinni, K. \& Meyer, J. (2001). Identification of archaeal rDNA from subgingival dental plaque by PCR amplification and sequence analysis. FEMS Microbiol Lett 196, 129-133.

Lepp, P. W., Brinig, M. M., Ouverney, C. C., Palm, K., Armitage, G. C. \& Relman, D. A. (2004). Methanogenic Archaea and human periodontal disease. Proc Natl Acad Sci U S A 101, 6176-6181.

Munson, M. A., Pitt-Ford, T., Chong, B., Weightman, A. \& Wade, W. G. (2002). Molecular and cultural analysis of the microflora associated with endodontic infections. J Dent Res 81, 761-766 (errata in 82, 69; 82, 247). 
Paster, B. J., Boches, S. K., Galvin, J. L., Ericson, R. E., Lau, C. N., Levanos, V. A., Sahasrabudhe, A. \& Dewhirst, F. E. (2001). Bacterial diversity in human subgingival plaque. J Bacteriol 183, 3770-3783.

Rocas, I. N. \& Siqueira, J. F., Jr (2005). Detection of novel oral species and phylotypes in symptomatic endodontic infections including abscesses. FEMS Microbiol Lett 250, 279-285.

Saito, D., Leonardo Rde, T., Rodrigues, J. L., Tsai, S. M., Hofling, J. F. \& Goncalves, R. B. (2006). Identification of bacteria in endodontic infections by sequence analysis of $16 \mathrm{~S}$ rDNA clone libraries. J Med Microbiol 55, 101-107.

Sakamoto, M., Rocas, I. N., Siqueira, J. F., Jr \& Benno, Y. (2006). Molecular analysis of bacteria in asymptomatic and symptomatic endodontic infections. Oral Microbiol Immunol 21, 112-122.

Sambrook, J., Fritsch, E. F. \& Maniatis, T. (1989). Molecular Cloning: a Laboratory Manual, 2nd edn. Cold Spring Harbor, NY: Cold Spring Harbor Laboratory.

Sedgley, C., Nagel, A., Dahlen, G., Reit, C. \& Molander, A. (2006). Real-time quantitative polymerase chain reaction and culture analyses of Enterococcus faecalis in root canals. J Endod 32, 173-177.
Siqueira, J. F., Jr \& Rocas, I. N. (2005). Exploiting molecular methods to explore endodontic infections: part 2 - redefining the endodontic microbiota. J Endod 31, 488-498.

Siqueira, J. F., Jr, Rocas, I. N., Baumgartner, J. C. \& Xia, T. (2005). Searching for Archaea in infections of endodontic origin. J Endod 31, 719-722.

Sundqvist, G. \& Figdor, D. (2003). Life as an endodontic pathogen: ecological differences between the untreated and root filled root canals. Endodontic Topics 6, 3-28.

Tran, S. D. \& Rudney, J. D. (1999). Improved multiplex PCR using conserved and species-specific $16 \mathrm{~S}$ rRNA gene primers for simultaneous detection of Actinobacillus actinomycetemcomitans, Bacteroides forsythus, and Porphyromonas gingivalis. J Clin Microbiol 37, 3504-3508.

Vianna, M. E., Conrads, G., Gomes, B. P. \& Horz, H. P. (2006). Identification and quantification of archaea involved in primary endodontic infections. J Clin Microbiol 44, 1274-1282.

Wade, W. G., Spratt, D. A., Dymock, D. \& Weightman, A. J. (1997). Molecular detection of novel anaerobic species in dentoalveolar abscesses. Clin Infect Dis 25 (suppl. 2), S235-S236. 